\title{
Types of Cancers Diagnosed and the Preference of Families of Adult Patients with Cancer about Disclosing Diagnosis to the Patients
}

\author{
Engida Abebe $^{1 *}$, Hana Abebe ${ }^{2}$
}

\author{
OPEN ACCESS \\ Citation: Engida Abebe, Hana Abebe: \\ Types of Cancers Diagnosed and the \\ Preference of Families of Adult Patients \\ with Cancer about Disclosing Diagnosis \\ to the Patient. J Health Sci \\ 2017;27(3):255. doi: \\ http://dx.doi.org/10.4314/ejhs.v27i1.7 \\ Received: November 28, 2016 \\ Accepted December 3, 2016 \\ Published: May 1, 2017 \\ Copyright: (c) 2017 Engdida A., et al. \\ This is an open access article distributed \\ under the terms of the Creative Commons \\ Attribution License, which permits \\ unrestricted use, distribution, and \\ reproduction in any medium, provided the \\ original author and source are credited. \\ Funding: Nil \\ Competing Interests: The authors \\ declare that this manuscript was approved \\ by all authors in its form and that no \\ competing interest exists. \\ Affiliation and Correspondence: \\ ${ }_{1,2}$ Department of surgery, SPHMMC , \\ Addis Ababa, Ethiopia, \\ "Email: engidaabebe@yahoo.com
}

\begin{abstract}
BACKGROUND: Cancer has become one of the top causes of death in developing nations killing more people than the common infectious diseases do. For several reasons, disclosing cancer diagnosis to the patient is a challenging job for physicians and family members.

MATERIALS AND METHODS: A cross-sectional study was done to determine the common cancer diagnosis and the preference about disclosing cancer diagnosis to the patients among attendants of adult cancer patients seen at the regular surgical OPD of St. Paul's Hospital Millennium Medical college (SPHMMC) in June 1-31st 2015. Medical records of the patients and face-to-face interview with attendants were used to generate the data and analysis was done with SPSS version 19.0.

RESULTS: A total 112(7.3\%) patients were diagnosed to have cancer and 104 attendants (93\%) were interviewed. The mean age of the patients was 48.2 years, Females made up $59 \%$ of the patients. The commonest cancer diagnosed was breast cancer. Male (62.7\%) and children (36.1\%) were the main attendants. Only $56.6 \%$ of the attendants agreed that patients should be the first to know diagnosis results. When possible, $84.3 \%$ preferred to hide diagnosis. Although $81.3 \%$ attendants did not like diagnosis disclosure to the patient, all of them wanted to know the diagnosis if they develop cancer. Nearly all, $98.8 \%$, of the attendants preferred to hear the diagnosis from their doctors.
\end{abstract}

CONCLUSIONS: Cancer is a relatively common diagnosis occurring at a younger age. The rate of cancer diagnosis disclosure acceptance and practice by attendants was low. Population-based and multicentre study with a larger sample size is recommended to define the condition better

KEYWORDS: Cancer, disclosure, reason 


\section{INTRODUCTION}

Cancer has become the second most common cause of death for human kind in the developed world. It was considered in the past to be a rare or less important problem in developing nations like Ethiopia. Now, cancer is one of the leading causes of death in developing countries, killing more people globally each year than AIDS, tuberculosis or malaria. More than half of all new cancer cases and over $60 \%$ of the deaths occur in the poorer regions of the world $(1,2)$. By 2020 , there will be 15 million new cases of cancer every year globally, $70 \%$ of which will be in developing countries (3).

It is a well understood fact that effective communication between physicians and patients and family is essential for the continuation of successful treatment that aims to improve survival, quality of life, or reduces psychological distress $(4,5)$. That is why effective doctor-patient communication is an integral part of good clinical care in general and in cancer specifically in particular (6). Communication can be seen as the main ingredient in medical care. Three different purposes of communication are identified, namely: (a) creating a good inter-personal relationship; (b) exchanging information; and (c) making treatment-related decisions (7).

Although it is something doctors do every day, communication to patients and family is not an easy job. Most physicians regard the communication of bad news such as cancer and chronic illnesses like end stage renal failure diagnosis as a difficult issue in clinical practice. As cancer is considered incurable and a killer with relatively shorter survival, it is the last thing patients and family want to hear as a diagnosis. The optimal manner of communicating bad news to patients and family so that physicians can create maximal understanding and facilitate their psychological adjustment is unknown (8). Continuing controversy surrounds the extent to which cancer patients should be informed of their diagnosis and its implications (5). A number of articles have provided advice for breaking bad news; however, guidelines differ considerably from one author to the other (9). Little is known about the effect of disclosing cancer diagnosis to patients on psychosocial makeup of attendants and the patient.

Communication, including disclosure of diagnosis, is one of the most difficult issues for doctors in cancer care $(6,10)$. This is complicated when it comes to a society where families have strong attachment and are involved in decision making not only in treatment but also in disclosing diagnosis (11). In developing nations like Ethiopia, extended family with strong bond and attachment within it is common. This can make communication to patients and family members a tougher job. When it comes to disclosure of the diagnosis, it is even worse (11). It is a common encounter to surgeons and physicians to be requested by family members, especially young attendants caring for parents, not tell a cancer diagnosis to patients. For such reasons and fear of telling the patient explicitly the diagnosis doctors tried other similar words by avoiding the word cancer (12).

Family members also try to avoid the term cancer while trying to inform the illness to the loved ones. In our clinical practice, we often witness attendants trying to hide the diagnosis of cancer from their family members or relatives (4, 5) Physicians in both the developing and developed world often time do not know whom to communicate first, what level of information patients and families needs, etc. The objective of this study was to determine common cancer diagnosis and preference of attendants of adult cancer patients regarding disclosing diagnosis of cancer to the patients.

\section{MATERIALS AND METHODS}

Study area and period: A cross-sectional study was done to determine the preference of attendants of adult cancer patients at the regular surgical OPD of SPHMMC, Addis Ababa, Ethiopia. St. Paul's hospital is the second big hospital in Addis Ababa (AA) which serves as a referral center for patients from the city and all over the country. The regular surgical OPD is the entry point for nonemergency surgical patients. There were three regular surgical OPDs where residents see all surgical referrals and investigate and transfer to

DOI: http://dx.doi.org/10.4314/ejhs.v27i3.7 
surgical referral clinics where patients are seen by consultant surgeons. All adult cancer patients and their primary attendants who showed up in June 1$31^{\text {st }} 2015$ were included in the study. Data was collected by trained surgical residents and general practitioners (who were working at the OPD) using a pre-tested structured questionnaire. Medical records of the patients were used for clinical data about the cancers and sociodemography of the patients. Attendants were interviewed face-to-face. The data collection process was supervised by the investigators. Data was cleaned, coded and entered and analyzed with SPSS version 19.0. Chi square test was used to test the presence of associations. Associations with $\mathrm{p}$-value $<0.05$ were considered significant. Ethical clearance was obtained from SPHMMC ethical review board.

\section{RESULTS}

Socio-demographic characteristics of the patients: A total of 1534 patients were seen at the regular surgical outpatient department on June 2015. Among these 112(7.3\%) patients were diagnosed to have cancer and attendants of $104(93 \%)$ patients were interviewed. The mean age of the patients was 48.2 years, but it ranged from 18 to 66 years. The age group of 40 to 60 years made the major share, $42.2 \%$. Patients below 20 years and above 60 years constituted $4.8 \%$ and $28.9 \%$ respectively. Females made up of $59 \%$ of the patients. The common cancer diagnoses were breast cancers $(26.5 \%)$, colorectal cancers $(19.3 \%)$ and thyroid neoplasms (13.3\%). As shown in Table 1, at presentations, the majority of the cancers were stage III $(57.8 \%)$ and stage IV $(18.1 \%)$ (Figure).

Table 1: Types and frequency of cancers diagnosed at SPHMMC regular OPD, June 2015.

\begin{tabular}{lllllll}
\hline & Males & & Females & Total & \\
\cline { 2 - 7 } Type of cancer & No. & $\%$ & No. & $\%$ & No. & $\%$ \\
Breast & 1 & 2.3 & 27 & 44.3 & 28 & 26.5 \\
Colorectal & 9 & 20.9 & 11 & 18 & 20 & 19.3 \\
Thyroid & 4 & 9.3 & 10 & 16.4 & 14 & 13.3 \\
Hepatobiliary & 8 & 18.6 & 3 & 4.9 & 11 & 10.8 \\
Gastric & 3 & 7 & 4 & 6.6 & 7 & 7.2 \\
Musculoskeletal & 3 & 7 & 2 & 3.3 & 5 & 4.8 \\
Genitourinary & 3 & 7 & 2 & 3.3 & 5 & 3.6 \\
Esophageal & 3 & 7 & 1 & 1.6 & 4 & 10 \\
Others & 9 & 20.9 & 1 & 1.6 & & 9.6 \\
\hline
\end{tabular}

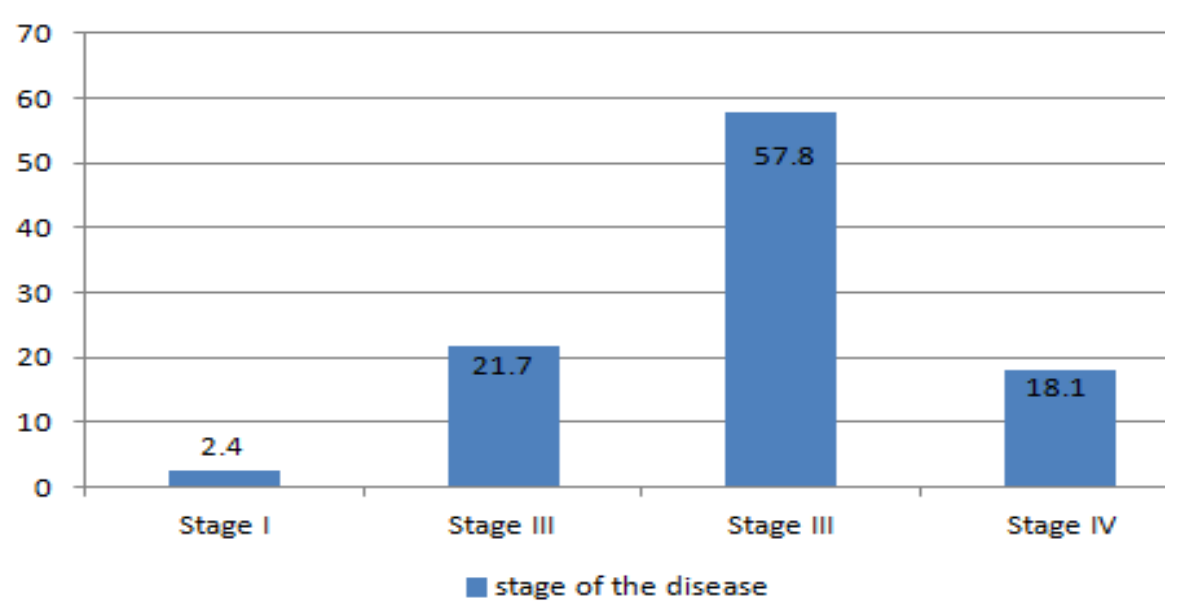

Figure 1: Stages of the cancers at presentation among patients diagnosed to have cancer at SPHMMC regular OPD, June 2015 
The attendants: The mean age of the attendants was 39.7 with a range of $26-52$ years. Concerning the age distribution, $50.6 \%$ were between $20-40$ years, $38.6 \%$ between $40-60$ years and $10.8 \%$ above 60 years. Among the attendants, $62.7 \%$ were male. A child was the attendant in $36.1 \%$ of the patients while siblings made up of $27.7 \%$ of the attendants. With regard, to religion, 55.4\% were Orthodox Christians, 28.9\% Muslims 15.7\% Protestants (Table 2).

Table 2: Relationship of attendants to the cancer patients, SPHMMC regular OPD, June 2015.

\begin{tabular}{lll}
\hline Relationship of the attendant to the patient & Frequency & Percent $(\%)$ \\
\hline Child & 36 & 36.1 \\
Sibling & 29 & 27.7 \\
Spouse & 11 & 10.8 \\
Cousin & 8 & 7.5 \\
Mother & 4 & 3.6 \\
Father & 4 & 3.4 \\
Friend & 3 & 2.4 \\
Neighbor & 3 & 2.4 \\
Others & 6 & 6.1 \\
\hline
\end{tabular}

The majority of the attendants (68\%) completed a high school or were college graduates. Urban residents made up $57.8 \%$ of the attendants. Regarding the average monthly income, $42.2 \%$ had an income of less than 2000 Birr (100 USD), $51.8 \%$ had between 2000 Birr and 5000 Birr (250 USD) while $6 \%$ were in the income group of greater than 5000 Birr per month (Table 3).

Table 3: Socio-demographic characteristics of attendants of cancer patients, SPHMMC, June 2015.

\begin{tabular}{lllll}
\hline & Socio demographic data & Frequency & Percent (\%) & P value \\
\hline Educational status & Illiterate & 11 & 10.8 & \\
& Grade 6 completed & 21 & 20.5 & 0.27 \\
& Grade 12 completed & 44 & 42.2 & \\
Average monthly & College graduate & 28 & 26.5 & 0.014 \\
income & $<$ 2000 birr & 44 & 42.2 & \\
Religion & 2000-5000 birr & 54 & 51.8 & 0.091 \\
& $>$ 5000 birr & 6 & 6 & \\
\multirow{2}{*}{ Place of residence } & Orthodox & 58 & 55.4 & 0.072 \\
& Protestant & 16 & 15.7 & \\
\hline
\end{tabular}

Cancer knowledge: Among the attendants, $89.2 \%$ stated that they had heard about cancer. Among these, $95.2 \%$ of claimed that they did not have sufficient knowledge about cancer. Regarding curability, $39.4 \%$ believed that some cancers are curable $(\mathrm{p}=0.000)$. Only $70.4 \%$ of the attendants said treatment is available within the country, and 91.5\% stated the treatment for cancer is costly.
Friends/relatives (75.6\%) and media (64.1\%) were their main source of information regarding cancer. Disclosure: Among the attendants, 56.6\% of them agreed that patients should be the first to know their diagnoses $(p=0.000)$ while $10.8 \%$ stated that patients should not know the diagnosis at all times (0.001). When possible, $84.3 \%$ stated that diagnosis should be hidden from the patients.

DOI: http://dx.doi.org/10.4314/ejhs.v27i3.7 
When asked who should be the first to know the diagnosis, $94 \%$ said the spouse while $6 \%$ of them preferred adult daughter/son. Two-fifth of the attendants $(39.8 \%)$ hid the diagnosis from their relatives for the following reasons: patient will be hopeless $(78.8 \%)$, patient will not tolerate the stress $(63.3 \%)$, it is incurable disease and there is no need for the patient to know about it (51.5\%). Among these attendants, $81.3 \%$ claimed that they would not like it but accept it if the diagnosis is disclosed directly to the patient, $9.7 \%$ stated that they would get angry, and $9.7 \%$ indicated that they would only be disappointed.

For the attendants who disclosed the diagnosis $(60.1 \%)$ for their relatives the following were their reasons: treatment will be difficult if the patient does not know the diagnosis (92\%), the patient has the right to know the diagnosis $(86 \%)$, the patient should know the treatment plan (16\%) and economic reasons (10\%).

All the attendants wanted to know the diagnosis if they developed cancers themselves. Among these, $96.4 \%$ would like to be the first to hear the diagnosis. During disclosure, $73.2 \%$ preferred to be told alone while $26.8 \%$ preferred to be with a family member. Among the attendants, 7.3\% preferred that the diagnosis be told for families first. Nearly all, $98.8 \%$ of them, preferred to hear the diagnosis from the doctor while $1.2 \%$ chose to hear from a family member.

Table 4: Reasons for disclosing or not disclosing cancer diagnosis by attendants of cancer patients, SPHMMC regular OPD, June 2015.

\begin{tabular}{llll}
\hline Reasons for disclosing diagnosis & $\%$ & Reason for not disclosing & $\%$ \\
\hline treatment will be difficult if the patient does & & patient will be hopeless & 78.8 \\
not know the diagnosis & 92 & & \\
patient has the right to know the diagnosis & 86 & patient will not tolerate the stress & 63.3 \\
want patient to share treatment plan & 16 & it is incurable disease & 51.5 \\
economic reasons & 10 & & \\
\hline
\end{tabular}

\section{DISCUSSION}

The cancers: The finding of a $7.3 \%$ of cancer incidence in this study, though may be an underestimate, is significant. It is not possible to compare this figure with a national level figure due to lack of cancer registry in Ethiopia. The figure is slightly less than the global cancer estimate for East African citizens' cumulative risk of developing cancer which is $9.4 \%$ for males and $11.3 \%$ for females (2). Our finding is lower because it focused on only patients who came to the hospital not what it might look in the society. Clinical experiences show that cancer is a significant health problem with an escalating incidence. For instance, at Tikur Anbessa Hospital, Addis Ababa, Ethiopia, the number of patients who visited the Radiotherapy and Oncology Center increased markedly through the years 1998-2011(2). Our finding showed the incidence of cancers to be higher in females. This could be due to high incidence of breast cancers in
Ethiopia which is the most common cancer diagnosed in women (13). This finding is in line with the global cancer estimate findings which showed that more than half of new cases of cancer in East Africa occurred in females $(170,500$ $(59.3 \%)$ out of 287,300$)$ in 2012.

Globally, the three most commonly diagnosed cancers in 2012 were lung, prostate and colorectal cancers among males, and breast, colorectal and lung cancers among females. In economically developing countries, the three most commonly diagnosed cancers were lung, liver and stomach in males, and breast, cervical and lung in females (14). In our study, colorectal cancer was found to be the leading cancer in males followed by hepatobiliary and thyroid cancer unlike the study from Tikur Anbessa Specialized Hospital in which Gastrointestinal malignancies took the third place in frequency (15). Unlike the global figures, lung cancer was not among the top cancers, probably due to lower rate of cigarette smoking in Ethiopian and because patients may be referred to

DOI: http://dx.doi.org/10.4314/ejhs.v27i3.7 
other hospitals in town (16). Prostatic cancer was not also among the leading cancers in this study. This can be explained by the relatively younger age of the study population. In this study, the most common malignancy in females was breast cancer followed by colorectal cancer and thyroid cancer. This was also shown in the study from Tikur Anbessa specialized Hospital (15).

Cancer occurs in a relatively younger age in developing countries. This was also the finding in our study, where $71 \%$ of the cancer patients were less than 60 years of age. Similar findings were also shown from Tikur Anbessa Specialized Hospital (15). The majority of cancers in Africa are diagnosed at an advanced stage because of lack of screening, early detection services and limited awareness of early signs and symptoms of cancer among the public and healthcare providers (14).This is also reflected in our study and a study from Tikur Anbessa Specialized Hospital in which early stage cancers, stage I or II, made less than $10 \%$ of the cancers (15).

The attendants: About half of the attendants were between the ages of 20-40 years. Males constituted two-thirds of the attendants. As most of the attendants were children of the patient, the relatively young attendants can be understood. The higher male attendants can also be understood as one recognizes that male is the economic backbone of Ethiopian societies engaged in family support at home and outside. Like in most developing nations, Ethiopian families are intimately attached. This fact was also seen in this study as the majority of the attendants $(70.8 \%)$ were first degree relatives of the patients (17).

The educational attainment of the attendants in this study was significantly higher compared to national figures. According to the Ethiopian demographic and health survey (EDHS) 2014 report, $48 \%$ females and $37 \%$ males were illiterate compared to our study population where only $14.7 \%$ male and $8.2 \%$ female attendants were illiterate (17).

Again, only $7.6 \%$ of the population had completed secondary or higher education nationally which is significantly lower than our study finding of $68 \%$ (17). This may be explained by the fact that the majority of the attendants were from urban areas, mainly Addis Ababa, where children are likely to go to school. This might partly explain why the majority of the attendants preferred to know their diagnosis if they develop cancer.

Despite having a relatively higher educational status, the main sources of information about cancer for the attendants were friends and relatives. One can understand that information from such group of the society can be incomplete, misunderstood and may result in spread of various misconceptions about cancer which can result in stigma and its consequences. Using primary school and secondary schools for teaching basic facts about cancer to adolescents and young adults can be used to increase facts about cancer and its consequences.

This work is probably the first of its kind, not only in Ethiopia but also in Africa, to investigate the perspectives of families of cancer patients in disclosing cancer diagnosis. There is only little information elsewhere too. This made comparison of our finding with other studies difficult. The authors strongly believe that patient should know his/her cancer diagnosis completely so that evaluation and treatment decision will be effective. It is a very common encounter those young family members (mostly children) asking not tell cancer diagnosis to their loved ones. In a study done in Turkey to assess factors affecting relatives' attitude towards honest disclosure of cancer, $66 \%$ of the relatives did not want to disclose the diagnosis which is slightly higher compared to our finding of $60 \%$ (11).The reasons for not disclosing in the above study were the fear that the patients would be extremely upset (57.3\%) and the patient would not want it $(29.3 \%)$. The remaining $13.4 \%$ could not give any reason for refusing disclosure (11). Regarding sociodemographic characters, the majority of the patients were from the low to medium income group (76\%) which is comparable to our study. The fact that patient sex plus type and stage of cancer were not associated with disclosure in our study was not in line with the findings of this Turkish study where male sex, non-breast cancer and stage IV disease were significantly associated with non-disclosure of cancer diagnosis (11).

DOI: http://dx.doi.org/10.4314/ejhs.v27i3.7 
Cancer continues to carry a significant amount of stigma obviously related to level of awareness of the condition. Although not reported by any of the attendants, stigma to cancer can be one reason why they preferred that the loved ones should not know their diagnosis. The nature of the disease does not make the stigma associated with it overtly felt, but the silence in it speaks loud. The stigma can be related to the perception that cancer and its treatment cause significant morbidity and mortality, or it could be considered as a divine punishment. Communication both to the patient and the family/attendants is critical to decreasing cancer-related stigma, raising cancer awareness, and disseminating cancer education.

Though attendants approval rate of patient should be the first to know his/her diagnosis is low $(56.6 \%)$, the majority of the them $(96.4 \%)$ wanted to be the first to know their diagnosis. This might indicate some transition of social structure (higher education attainment) or values and could be one area to investigate continuously. Among family members, spouses were chosen to be told the diagnosis of cancer first by a great majority of the attendants although most attendants were children. This indicates that asking the spouse to come to the hospital during disclosure of diagnosis should be considered.

A great proportion of the attendants preferred to be told the diagnosis of cancer alone if they developed cancer themselves probably to reduce the psychological stress on family members. Nearly all of the attendants preferred to hear the diagnosis from the doctors, possibly to obtain detailed and accurate information about their illnesses.

In conclusion, cancer is a relatively common diagnosis occurring relatively at a younger age in Ethiopia. Children were the main attendants of patients who were diagnosed with cancer. The rate of cancer diagnosis disclosure acceptance and practice by attendants was low. The knowledge that cancer is curable and the treatment is available within the country was associated with a higher disclosure rate. Physicians need to understand both the perspective of patients and the attending family members in disclosing cancer diagnosis. Because little is known about this issue in Ethiopia, multicentre study with a larger sample size is recommended.

\section{REFERENCES}

1. Garcia M, Jemal A, Ward EM, Center MM, Hao Y, Siegel RL, Thun MJ.Global Cancer Facts \& Figures 2007. Atlanta, GA: American Cancer Society, 2007.

2. D. Maxwell Parkin, Freddie Bray, Jacques Ferlay and Paola Pisani. Estimating the World Cancer Burden: GLOBOCAN 2000. Int. J. Cancer 2001; 94:153-156.

3. P. Kanavos; The rising burden of cancer in the developing world. Ann Oncol 2006; 17 (suppl_8): viii15-viii23.

4. Butow PN, Tattersall MH, Goldstein D. Communication With Cancer Patients In Culturally Diverse Societies. Ann N Y Acad Sci. 1997; 809:317-329.

5. Bozcuk H, Erdog an V, Eken C, Plak E, Samur M, Zdogan M, Savas B.Does awareness of the diagnosis make any difference in quality of life? Determinants of emotional functioning in a group of cancer patients in Turkey. Support Care Cancer. 2002; 10:51-57.

6. Michael Bennett, Dawn Alison. Discussing the diagnosis and prognosis with cancer patients. Postgrad MedJ. 1996; 72: 25-29.

7. L M. L. Ong, I. J. C. J. M. DE Haes, 1 A. M. Hoos $\mathrm{j}$ and F. B. LAMMES. DoctorPatient Communication: A Review of the Literature. Soc. Sci. Med. 1995; 40(7):903918).

8. Maiko Fujimoriand Yosuke Uchitomi. Preferences of Cancer Patients Regarding Communication of Bad News: A Systematic Literature Review. Jpn J Clin Oncol. 2009;39(4):201-216.

9. Lesley Fallowfield, Valerie Jenkins. Communicating sad, bad, and difficult news in medicine. Lancet 2004; 363: 312-19.

10. Phyllis N. BUtOW, John N. Kazemi,Linda J. Beeney and et al. When the Diagnosis Is Cancer; Patient Communication Experiences and Preferences. Cancer. 1996; 72(12):26307. 
11. Mustafa Ozdogan, Mustafa Samur,Hakan Sat Bozcuk ,and et al . "Do not tell": what factors affect relative'sattitudes to honest disclosure of diagnosis to cancer patients? Support Care Cancer. 2004 12:497-502.

12. Stewart M. Dunn, Patricia U. Patterson, Phyllis N. Butow, and et al. Cancer by another Name: A Randomized Trial of the Effects of Euphemism and Uncertainty in Communicating With Cancer Patients. J. Clin. Oncol. 1993; 11(5): 989-996.

13. Yohannes W Woldeamanuel,Belaineh Girma, Alula M Teklu. Cancer in Ethiopia. Lancet Oncol. 2013;14(4):289-90.

14. American Cancer Society. Global Cancer Facts \& Figures 3rd Edition. Atlanta: American Cancer Society; 2015.
15. Tigeneh W. Pattern of Cancer in Tikur Anbessa Specialized Hospital Oncology Center in Ethiopia from 1998 to 2010. Int J Cancer Res Mol Mech. 2015;1(1): doi http://dx.doi.org/10.16966/ ijcrmm.103

16. Who Global Report on Trends in Prevalence of Tobacco Smoking 2015. Geneva. World Health Organization

17. Central Statistical Agency (CSA) [Ethiopia] and ICF. 2016. Ethiopia Demographic and Health Survey 2016:Key

18. Indicators Report. Addis Ababa, Ethiopia, and Rockville, Maryland, USA. CSA and ICF.

19. Daher M. Cultural beliefs and values in cancer patients. Ann Oncol. 2012; 23(3):66-9. 